\title{
GMR
}

\section{Prognostic significance of tumor-associated macrophage infiltration in gastric cancer: a meta-analysis}

\author{
X.L. Wang', J.T. Jiang ${ }^{1,2,3}$ and C.P. Wu ${ }^{1,2,3,4}$ \\ ${ }^{1}$ Department of Tumor Biological Treatment, \\ Third Affiliated Hospital of Soochow University, Changzhou, China \\ ${ }^{2}$ Jiangsu Engineering Research Center for Tumor Immunotherapy, \\ Changzhou, China \\ ${ }^{3}$ Institute of Cell Therapy, Soochow University, Changzhou, China \\ ${ }^{4}$ Department of Oncology, Third Affiliated Hospital of Soochow University, \\ Changzhou, China \\ Corresponding author: C.P. Wu \\ E-mail:wcpjjt@163.com
}

Genet. Mol. Res. 15 (4): gmr15049040

Received August 8, 2016

Accepted September 12, 2016

Published December 2, 2016

DOI http://dx.doi.org/10.4238/gmr15049040

Copyright (C) 2016 The Authors. This is an open-access article distributed under the terms of the Creative Commons Attribution ShareAlike (CC BY-SA) 4.0 License.

\begin{abstract}
Tumor-associated macrophages (TAMs), which play a crucial role in the tumor microenvironment, can be divided into M1 and M2 phenotypes, these phenotypes may exert opposite effects on the prognoses of patients with gastric cancer (GC). The association between TAMs and GC is contentious. Thus, a meta-analysis of 12 studies (incorporating 1388 patients) retrieved from the Cochrane Library, PubMed, and Embase databases was conducted in order to evaluate the relationship between TAMs and GC prognosis. Hazard ratios (HRs) with $95 \%$ confidence intervals (CIs) were pooled to explore the effect of these cells on survival of GC patients. Our results implied that high
\end{abstract}


total TAM infiltration levels correspond to worse overall survival (OS) in patients with $\mathrm{GC}(\mathrm{HR}=1.70,95 \% \mathrm{CI}=1.39-2.09 ; \mathrm{P}<0.001)$, and a similar result was observed in relation to $\mathrm{M} 2$ macrophage infiltration $(\mathrm{HR}=1.71,95 \% \mathrm{CI}=1.19-2.45 ; \mathrm{P}=0.004)$. In contrast, elevated $\mathrm{M} 1$ macrophage density in GC patients was associated with better OS (HR $=0.46,95 \% \mathrm{CI}=0.33-0.65 ; \mathrm{P}<0.001)$. This meta-analysis showed that the numbers of infiltrating M2 macrophages and total TAMs might be negative prognostic factors for patients with GC, while M1 macrophage infiltration may be associated with a favorable survival rate.

Key words: Tumor-associated macrophages; Gastric cancer; Prognosis; Meta-analysis

\section{INTRODUCTION}

Gastric cancer (GC) is a commonly occurring malignancy in China, and the second major cause of cancer deaths globally (Torre et al., 2015). Despite recent advances in diagnostic and treatment strategies, overall survival (OS) of GC patients remains poor, with a 5 -year rate of $4 \%$ for stage-IV cases (Thrumurthy et al., 2015). Immunotherapies such as adoptive cell transfer, cancer vaccines, and monoclonal antibody treatments offer new options for $\mathrm{GC}$ patients, as does the development of new combination therapies targeting different mechanisms involved in tumor progression.

Tumor-associated macrophages (TAMs) function as immune regulators and constitute potential targets in cancer immunotherapy (Ruffell and Coussens, 2015). TAMs may exert a dual effect on tumor growth, owing to the polarization of macrophages into two distinct phenotypes, M1 and M2 (Mantovani and Locati, 2013). M1 macrophages function in antitumor immunity, whereas M2 macrophages contribute to tumor progression (Murray and Wynn, 2011). Recently, Mills (2012) and Mills and Ley (2014) described M1 and M2 macrophages as 'inhibit' and 'heal' types, respectively, owing to their respective stimulation of the Th1 and Th2 response. M1 polarization is known as classical activation, and is considered to be induced by lipopolysaccharide and cytokines such as interferon- $\gamma$ and tumor necrosis factor- $\alpha$ (Mills, 2012; Biswas et al., 2013; Mantovani and Locati, 2013), whereas M2 macrophages are termed alternatively activated, and are thought to be induced by prostaglandin E2 and cytokines including interleukin (IL)4 and IL10 (Sica et al., 2008; Liu et al., 2012; Biswas et al., 2013). M1 macrophages promote Th1 responses with microbicidal and tumoricidal effects (Mills, 2012; Biswas et al., 2013; Mantovani and Locati, 2013), whereas M2 macrophages induce Th2 responses and promote tissue repair and remodeling, angiogenesis, immune suppression, and tumor progression (Sica et al., 2008; Biswas et al., 2013).

Although the dual roles of TAMs in tumor progression have been supported by studies both in vitro and in vivo using different tumor models, the effect of tumor-infiltrating TAMs and TAM subsets on GC prognosis remains controversial. This may be due to both the heterogeneity of TAM subsets and the functional plasticity of TAMs in the tumor microenvironment. A variety of markers (including CD68, CD163, CD206, etc.) have been used to identify different TAM subsets in tumor tissues (Ishigami et al., 2003; Ohno et al., 2005; Haas et al., 2009; Kawahara et al., 2010; Wang et al., 2011; Kim et al., 2015; Lin et al., 2015; Wu et al., 2015; Zhang et al., 2015; Park et al., 2016; Zhang et al., 2016). Among them, CD68 is commonly

Genetics and Molecular Research 15 (4): gmr15049040 
used as a cell surface marker of total infiltrating TAMs. Zhang et al. (2012) reported that a high density of such cells is associated with worse OS of patients with GC, not taking into account TAM subsets. Certain technical limitations apply to the conclusions drawn in previous studies, including small sample sizes restricting their statistical power. Therefore, we carried out the present meta-analysis to explore the prognostic significance of different TAM types in the GC tumor microenvironment, by pooling data from 12 eligible investigations.

\section{MATERIAL AND METHODS}

This analysis was performed according to the guidelines of the Preferred Reporting Items for Systematic Reviews and Meta-Analyses statement (Liberati et al., 2009).

\section{Search strategy}

Embase, PubMed, and the Cochrane Library (last update: April 19, 2016) were searched for studies having evaluated TAM density and survival in GC. The following terms were applied for study extraction: "tumor-associated macrophage OR TAMs OR tumor associated macrophage" (all fields) AND "gastric OR stomach" (all fields) AND "tumor OR tumour OR neoplasm OR cancer OR carcinoma" (all fields) AND "prognosis OR prognostic OR survival OR outcome" (all fields). Besides titles, abstracts, and full texts, references included in the extracted studies were also searched to identify potentially relevant articles.

\section{Inclusion and exclusion criteria}

Studies were selected using the following inclusion criteria: 1) articles concerning GC and the prognostic significance of TAMs or TAM polarization (M1 and M2 macrophages); 2) sufficient data were included for the estimation of hazard ratios (HRs) and 95\% confidence intervals (CIs); 3) macrophage infiltration in GC was categorized into high (above the cut-off value) and low (below the cut-off value) densities. The following exclusion criteria were also applied: 1) studies providing insufficient data for the calculation of HRs and 95\%CIs were excluded; 2) when two or more studies examined the same group of patients, only the latest or most comprehensive article was used; 3) case reports, animal trials, reviews, and conference abstracts were not included.

\section{Data extraction and quality assessment}

The following information was extracted: author's first name, year, source of study population, number of patients, tumor stage, follow-up information, detection method, TAM polarization, macrophage markers, cut-off value, HRs relating to the effect of TAMs and their polarization on OS and disease-free survival (DFS), and corresponding 95\%CIs. When both univariate and multivariate analyses were included in a study, only the latter was used. The quality of each article was evaluated according to the Newcastle-Ottawa Scale (NOS; Stang, 2010), scores for which vary from 0 to 9 . Eligible articles with scores $\geq 6$ were considered to be of high quality.

\section{Statistical analysis}

HRs with 95\%CIs were applied to explore the association between TAM density and

Genetics and Molecular Research 15 (4): gmr15049040 
GC patient prognosis, with an $\mathrm{HR}>1$ indicating worse prognosis, and an $\mathrm{HR}<1$ implying better prognosis. If such data were not directly stated in the article, they were extracted from available information following the guidelines set out by Tierney et al. (2007). When values of variables could only be taken from figures, data in Kaplan-Meier survival curves were extracted with Engauge Digitizer version 4.1 (free software downloaded from http:// sourceforge.net), which was used to reproduce HRs and their 95\%CIs. Statistical heterogeneity was tested graphically by the chi-square test, with a P value cut-off of 0.10 , and by calculating $I^{2}$ (Higgins and Thompson, 2002; Higgins et al., 2003). If a $\mathrm{P}$ value $<0.10$ or an $I^{2}$ value $>50 \%$ was returned, a random-effect model was applied to assess the data; otherwise, a fixed-effect model was used. Publication bias was evaluated with the Egger tests and Begg's funnel plots. All analyses were conducted in Stata 12.0 (StataCorp LP, College Station, TX, USA).

\section{RESULTS}

\section{Study characteristics}

In total, 125 records were initially retrieved from the databases. These potentially relevant articles were screened for eligibility based on duplication, title, abstract, and publication type, after which, 110 were excluded. The full texts of 15 candidate articles were carefully reviewed, with three being excluded as a result (two with inadequate data and one having examined the same patient group as another study). Finally, 12 studies were included in the meta-analysis (Figure 1; Ishigami et al., 2003; Ohno et al., 2005; Haas et al., 2009; Kawahara et al., 2010; Wang et al., 2011; Amoueian et al., 2015; Kim et al., 2015; Lin et al., 2015; Wu et al., 2015; Park et al., 2016; Zhang et al., 2015, 2016). In total, 1388 patients from China, Taiwan, Korea, Iran, Japan, and Germany were included. Four studies incorporated fewer than 100 patients, and three examined over 150 patients. Nine articles reported the prognostic value of total infiltrating TAMs (Ishigami et al., 2003; Ohno et al., 2005; Haas et al., 2009; Kawahara et al., 2010; Amoueian et al., 2015; Kim et al., 2015; Wu et al., 2015; Zhang et al., 2015, 2016), whereas others focused on specific TAM polarization subsets. Immunohistochemistry was used to detect both total infiltrating TAMs and TAM polarization. Cut-off values were chosen according to several measures, including the median $(\mathrm{N}=8)$ and mean $(\mathrm{N}=1)$. Other characteristics of the included articles are shown in Table 1. The average NOS score was 6.8 .

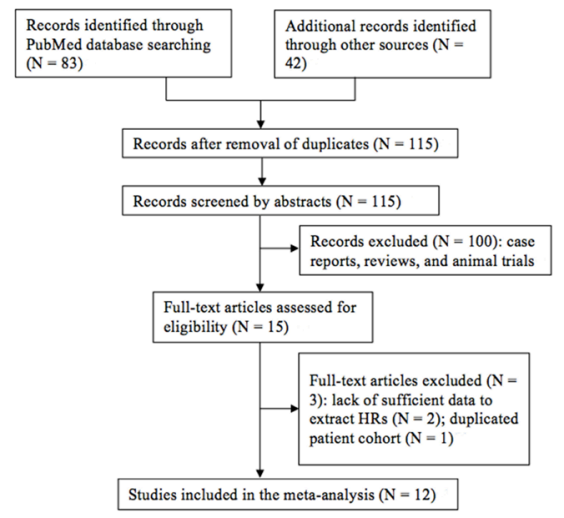

Figure 1. Flow chart outlining the selection of studies. Ultimately, 12 studies were selected to establish the association between tumor-associated macrophage density and gastric cancer prognosis. HR = hazard ratio.

Genetics and Molecular Research 15 (4): gmr15049040 
TAMs in gastric cancer: a meta-analysis

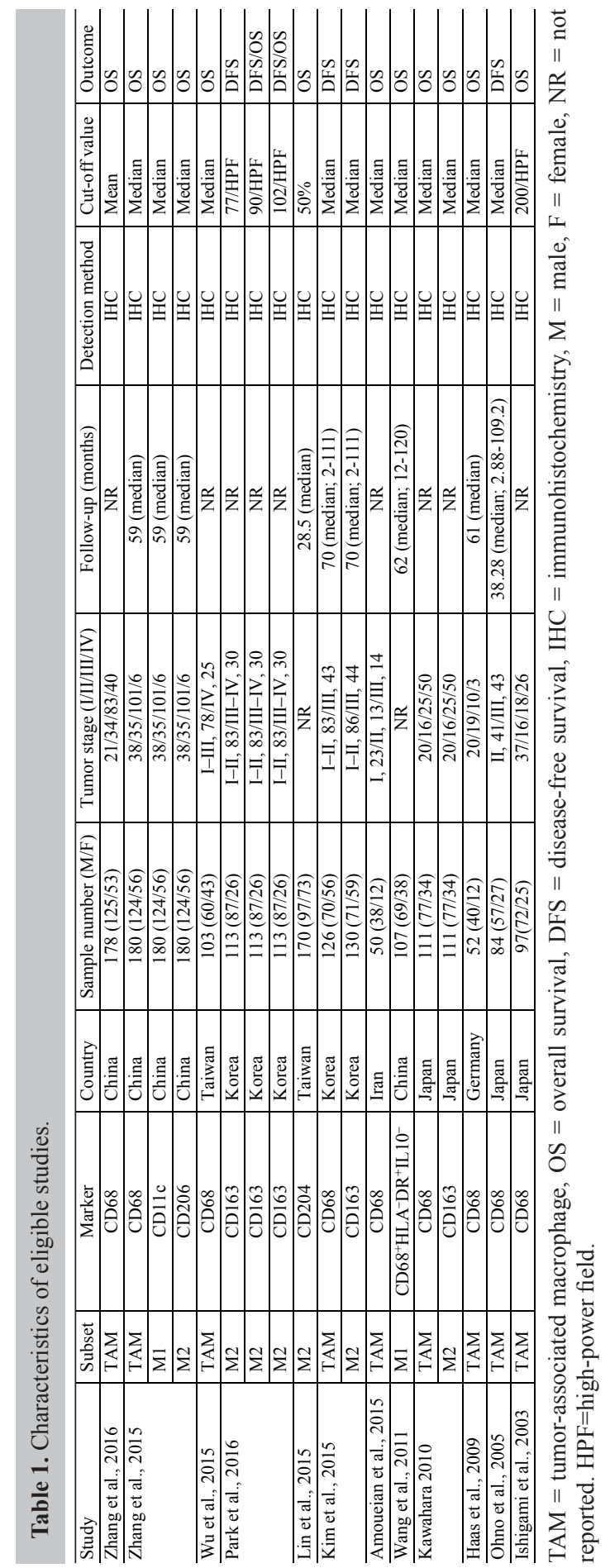

Genetics and Molecular Research 15 (4): gmr15049040 
CD68 was commonly used to identify macrophages, being employed as a TAM marker in 10 studies, one of which utilized it in combination with other specific macrophage markers. Zhang et al. (2015) and Wang et al. (2011) identified M1 macrophages as CD11 $\mathrm{c}^{+}$and $\mathrm{CD}^{+}{ }^{+} \mathrm{HLA}^{-} \mathrm{DR}^{+} \mathrm{IL}^{-} 0^{-}$cells, respectively. M2 macrophages were recognized as $\mathrm{CD} 163^{+}$cells in three studies (Kawahara et al., 2010; Kim et al., 2015; Park et al., 2016), and as CD206 ${ }^{+}$and CD204 ${ }^{+}$cells in one study each (Lin et al., 2015; Zhang et al., 2015).

\section{Subgroup analysis}

Outcomes of GC patients corresponded remarkably to TAM polarization status. Thus, we performed subgroup analyses based on this variable, the principal results of which are given in Table 2.

Table 2. Pooled hazard ratios concerning the relationship between tumor-associated macrophage polarization and prognosis of patients with gastric cancer.

\begin{tabular}{|c|c|c|c|c|c|c|c|c|c|}
\hline \multirow[t]{2}{*}{ Subset } & \multirow[t]{2}{*}{ Outcome } & \multirow{2}{*}{$\begin{array}{l}\text { No. of } \\
\text { studies }\end{array}$} & \multirow{2}{*}{$\begin{array}{l}\text { No. of } \\
\text { patients }\end{array}$} & \multirow[t]{2}{*}{ HR $(95 \% \mathrm{CI})$-model } & \multirow[t]{2}{*}{$P$} & \multicolumn{2}{|c|}{ Heterogeneity } & \multicolumn{2}{|c|}{ Publication bias } \\
\hline & & & & & & $I^{2}, \%$ & $\mathrm{P}$ & Begg's P & Egger's P \\
\hline \multirow{2}{*}{ Total TAMs } & OS & 7 & 771 & $1.70(1.39-2.09)$-fixed effects & $<0.001$ & 17.6 & 0.295 & 1.000 & 0.762 \\
\hline & DFS & 2 & 227 & $1.13(0.33-3.89)$-random effects & 0.846 & 68.1 & 0.076 & - & - \\
\hline M1 & OS & 2 & 287 & $0.46(0.33-0.65)$-fixed effects & $<0.001$ & 0 & 0.556 & - & - \\
\hline M2 & OS & 4 & 574 & 1.71 (1.19-2.45)-random effects & 0.004 & 71.2 & 0.015 & 0.734 & 0.052 \\
\hline
\end{tabular}

$\mathrm{HR}=$ hazard ratio, $\mathrm{CI}=$ confidence interval, $\mathrm{TAM}=$ tumor-associated macrophage, $\mathrm{OS}=$ overall survival, $\mathrm{DFS}=$ disease-free survival.

\section{Total infiltrating TAMs and GC prognosis}

Among the 12 included studies, nine reported the relationship between total infiltrating TAMs and patient survival. Seven of the articles evaluated OS (Ishigami et al., 2003; Haas et al., 2009; Kawahara et al., 2010; Amoueian et al., 2015; Wu et al., 2015; Zhang et al., 2015, 2016), and two recorded DFS (Ohno et al., 2005; Kim et al., 2015). The pooled HR of the former seven studies indicated that TAM infiltration correlates with worse OS (high $\mathrm{CD}^{+} 8^{+}$TAM density $v s$ low $\mathrm{CD} 68^{+} \mathrm{TAM}$ density, $\mathrm{HR}=1.70,95 \% \mathrm{CI}=1.39-2.09 ; \mathrm{P}<0.001$; heterogeneity: $I^{2}=17.6 \%, \mathrm{P}=0.295$; Figure $2 \mathrm{~A}$ ).

Two investigations focused on CD68 $8^{+}$TAM density and DFS (Ohno et al., 2005; Kim et al., 2015). A random-effects model was applied to estimate their pooled $\mathrm{HR}$ and its $95 \% \mathrm{CI}$ owing to the presence of significant heterogeneity $\left(\mathrm{P}=0.076, I^{2}=68.1 \%\right.$; Figure $\left.2 \mathrm{~B}\right)$. The result of our analysis implied that there is no association between high TAM levels and DFS in $\mathrm{GC}(\mathrm{HR}=1.13,95 \% \mathrm{CI}=0.33-3.89 ; \mathrm{P}=0.846)$.

\section{M1 macrophage density correlates with better OS}

Two studies investigated the relationship between M1 macrophages and patient survival (Wang et al., 2011; Zhang et al., 2015). Zhang et al. (2015) and Wang et al. (2011) considered $\mathrm{CD} 11 \mathrm{c}^{+}$and $\mathrm{CD}^{+} 8^{+} \mathrm{HLA}^{-} \mathrm{DR}^{+} \mathrm{IL} 10^{-}$cells to be $\mathrm{M} 1$ macrophages, respectively. Both are thought to be valid markers of the M1 macrophage subset. A fixed-effect model was applied to pool HRs, as no significant heterogeneity was detected $\left(\mathrm{P}=0.556, I^{2}=0.0 \%\right.$; Figure $3)$. High M1 macrophage density in GC tissues was found to be associated with favorable OS $(\mathrm{HR}=0.46,95 \% \mathrm{CI}=0.33-0.65 ; \mathrm{P}<0.001)$. 


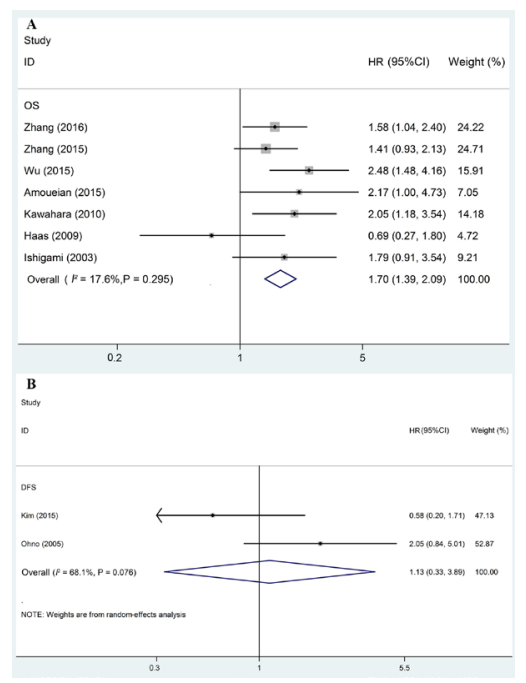

Figure 2. Forest plots of studies showing hazard ratios (HRs) relating to the influence of high levels of total infiltrating tumor-associated macrophages on gastric cancer. A. Forest plot of prognostic effect based on overall survival (OS). B. Forest plot of prognostic effect based on disease-free survival (DFS). CI = confidence interval.

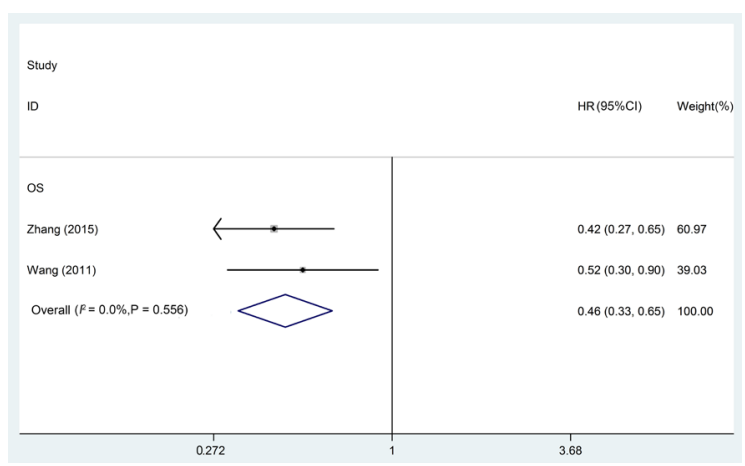

Figure 3. Forest plot of studies showing hazard ratios (HRs) relating to the influence of high levels of M1 macrophages on gastric cancer. $\mathrm{CI}=$ confidence interval.

\section{M2 macrophage subset correlates with worse OS}

Five studies assessed the connection between M2 macrophages and patient survival (Kawahara et al., 2010; Kim et al., 2015; Lin et al., 2015; Zhang et al., 2015; Park et al., 2016), four of which explored the relationship between these cells and OS. Kawahara et al. (2010) and Park et al. (2016) identified M2 macrophages as CD163+ cells, whereas Zhang et al. (2015) and Lin et al. (2015) considered them to be CD206 ${ }^{+}$and CD204 ${ }^{+}$cells, respectively. All three markers are believed to be able to indicate M2 macrophages. As significant heterogeneity was evident among these studies $\left(\mathrm{P}=0.015, I^{2}=71.2 \%\right.$; Figure 4$)$, overall estimates were calculated using a random-effect model, the result of which suggested that GC patients with high M2 macrophage densities exhibit worse OS $(\mathrm{HR}=1.71,95 \% \mathrm{CI}=1.19-2.45 ; \mathrm{P}=0.004)$.

Genetics and Molecular Research 15 (4): gmr15049040 


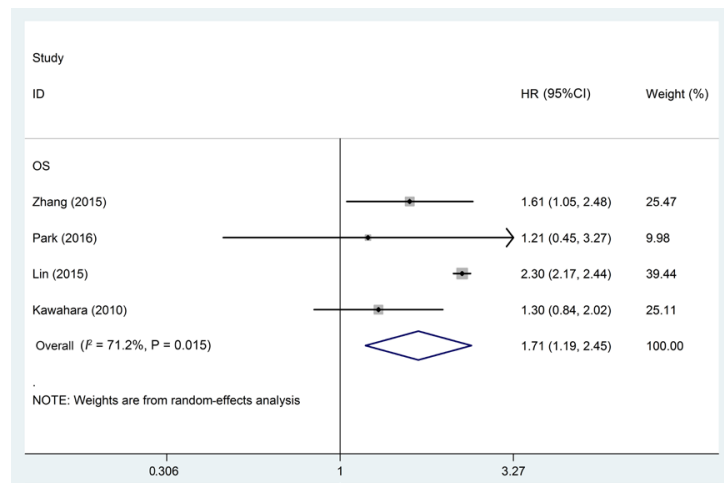

Figure 4. Forest plot of studies showing hazard ratios (HRs) relating to the influence of high levels of M2 macrophages on gastric cancer. $\mathrm{CI}=$ confidence interval.

\section{Sensitivity analysis demonstrated statistically insignificant impact of publication bias}

We conducted sensitivity analyses to determine whether any individual study substantially affected the pooled HRs. The extent of funnel plot asymmetry was estimated using Egger's test. This test of bias in the analysis of total infiltrating TAMs and OS returned a P value of 0.762 (Table 2). Concerning the M2 macrophage subset and OS, the Egger test gave a P value of 0.052 . To test the interdependence of effect size and variance, we carried out the Begg tests for each analysis. These demonstrated $\mathrm{P}$ values of 1.000 and 0.734 in regard to total infiltrating TAMs and OS and M2 macrophages and OS, respectively. Sensitivity analysis concerning our examination of total infiltrating TAMs and OS, conducted with a fixed-effect model, revealed that the result was not significantly affected by any individual study. P values of all Egger's and Begg's tests were greater than 0.05 , and funnel plots of analyses of total infiltrating TAMs and M2 macrophages were symmetric (Figure 5).
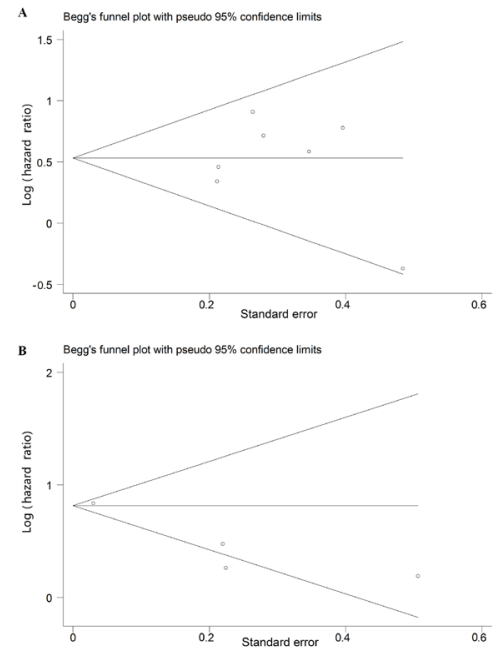

Figure 5. Begg's funnel plots concerning publication bias among the included studies. A. Total infiltrating tumorassociated macrophages and overall survival. B. M2 macrophages and overall survival. 
Therefore, no clear indication of publication bias was evident in our meta-analysis. Owing to the small number of studies included in the remaining two subgroup analyses, tests of publication bias were not performed.

\section{DISCUSSION}

TAMs constitute an important component of the tumor immune microenvironment, releasing many cytokines and promoting tumor progression via diverse mechanisms, including therapeutic resistance, angiogenesis, immune suppression, and metastasis (Ruffell and Coussens, 2015). The effect of TAMs has been observed to vary between solid tumor types in different studies performed using human specimens. However, recent investigations concerning TAMs in GC patients have generated conflicting data. This meta-analysis was performed to clarify the potential role of these cells in GC prognosis, by investigating the relationship between TAM infiltration and patient survival based on studies of GC tissues using different cell markers. Our results showed that high total infiltrating TAM density and M2 macrophage infiltration in GC were both associated with poor OS, while elevated M1 macrophage presence correlated with better OS. Moreover, there was no association between total infiltrating TAMs and DFS in GC patients.

A growing body of evidence indicates that the tumor microenvironment exerts a crucial influence on the determination of leukocyte function and phenotype. It is increasingly recognized that inflammation contributes to the development of many tumors. In combination with certain other cell types, activated macrophages are considered to be central to tumorassociated inflammation, indicating the involvement of host systems in tumor growth (Balkwill et al., 2005). It has been demonstrated that M1 and M2 TAMs have opposing effects on tumor growth, the latter being considered promotors of tumor expansion, and the former playing a protective role in such progression. The function of TAMs in cancer is not immutable, and is influenced by the tumor microenvironment. As TAMs are more closely associated with M2, rather than M1, macrophage characteristics, numerous articles have concluded that high TAM levels correspond to poor survival in many cancers, including those of the ovary, breast, lung, and endometrium (Zhang et al., 2013; Kübler et al., 2014; Pei et al., 2014; Reinartz et al., 2014; Yuan et al., 2014). Nevertheless, inconclusive findings have been published with respect to GC (Komohara et al., 2014). Such ambiguous results may be due to a failure to distinguish between $\mathrm{M} 1$ and $\mathrm{M} 2$ phenotypes.

Many studies have used CD68 to identify total infiltrating TAMs. Indeed, this is the most commonly utilized marker of these cells. Gottfried et al. (2008) investigated the expression of CD68 in different primary cultures and cancer cell lines, finding it to be widespread, including in fibroblasts, monocytes, macrophages, endothelial cells, and even certain cancer cells. CD68 may not be specific to TAMs, but merely enriched in this cell type (Gottfried et al., 2008). Therefore, it may nonetheless be a relatively reliable TAM marker. Nine of the 12 included studies used CD68 as a macrophage marker (Ishigami et al., 2003; Ohno et al., 2005; Haas et al., 2009; Kawahara et al., 2010; Amoueian et al., 2015; Kim et al., 2015; Wu et al., 2015; Zhang et al., 2015, 2016). The corresponding pooled HR indicated that high levels of $\mathrm{CD} 68^{+}$TAMs correlated with poor OS, but no significant correlation was identified between such cells and DFS.

Increasing evidence suggests that TAM infiltration can predict the OS of patients having undergone surgical resection for GC, although the specific underlying mechanisms

Genetics and Molecular Research 15 (4): gmr15049040 
remain unclear. It has been reported that TAMs can spread via lymphatic flow in the premetastatic lymph nodes of GC patients (Go et al., 2016), which is considered to be one of the mechanisms responsible for increased tumor cell invasiveness and metastasis. Zhang et al. (2016) found TAMs to be associated with the induction of epithelial-mesenchymal transition in human GC tissues. Moreover, Lin et al. (2015) revealed that the interaction between osteopontin and TAMs can promote GC progression. One study included in the present analysis revealed that TAM density is remarkably related to poor survival of patients with intestinal GC, whereas no such association is evident concerning those with diffuse GC (Kawahara et al., 2010), suggesting that the influence of TAMs on this disease's prognosis depends on histological type.

However, our study found no association between total infiltrating TAMs and DFS. First, this may be due to the limitations of our analysis. The number of included articles and the sample size were relatively small, resulting in the detection of significant heterogeneity among studies. Second, of the articles in this subgroup, Kim et al. (2015) investigated total infiltrating TAMs, whereas Ohno et al. (2005) focused on those localized to the invasive margin. TAMs in different locations may have divergent effects on tumor growth and immunosuppression. Ohno et al. (2005) concluded that TAMs in the invasive margin promote tumor growth by suppressing the immune response. Third, DFS refers to the period of time after primary treatment during which no signs or symptoms of cancer are observed, whereas OS is measured until the death of the patient. The tumor microenvironment may change during tumor progression, altering the functions of total infiltrating TAMs. Moreover, Park et al. (2016) reported that high M2 macrophage infiltration correlates with poor DFS, while Kim et al. (2015) showed that elevated densities of such cells are associated with improved DFS. These contrasting results indicate that the relationship between polarized TAMs and DFS remains controversial, which may partially influence the observed effect of total infiltrating TAMs.

Various cytokines can activate M1 macrophages, which go on to produce proinflammatory molecules. M2 macrophages release factors that advance tumor progression, promote angiogenesis, and adjust adaptive immunity to contribute to metastasis (Biswas and Mantovani, 2010). CD11 $\mathrm{c}^{+}$and $\mathrm{CD}^{+} 8^{+} \mathrm{HLA}^{-} \mathrm{DR}^{+} \mathrm{IL} 10^{-}$cells, both of which are considered representative of $\mathrm{M} 1$ macrophages, were used in studies included in the present meta-analysis (Wang et al., 2011; Zhang et al., 2015). A number of studies focused on tumor-promoting M2 macrophages using more specific markers, including CD206, CD204, and CD163 (Kawahara et al., 2010; Lin et al., 2015; Zhang et al., 2015; Park et al., 2016). The latter functions as a macrophage-associated scavenger receptor (Nam et al., 2014), and is known to be an efficient marker distinguishing M2 macrophages from other cell types. CD206 and CD204 were also used as markers in investigations incorporated into our analysis (Lin et al., 2015; Zhang et al., 2015). As markers of M2 macrophages, these molecules are considered to be as effective as CD163. Our results imply that high levels of M2 macrophages are related to poor survival. This may be partly explained by the fact that chronic inflammation is closely associated with gastric tumors, in which M2 macrophages can be observed after staining for the appropriate markers. Park et al. (2016) reported that $\mathrm{CD} 163^{+}$TAMs are linked to increased microvessel density, suggesting that M2 macrophages facilitate angiogenesis in GC patients. Although the pooled HR of these studies confirmed an association with M2 macrophages, the prognostic roles of different markers of such cells remain to be investigated.

The degree of TAM infiltration may be utilized as a prognostic factor, and could even serve as a therapeutic target in GC. Furthermore, the prognostic influence of polarized TAMs can be combined with tumor-node-metastasis staging to estimate GC patient prognosis, guiding

Genetics and Molecular Research 15 (4): gmr15049040 
clinicians in the formulation of improved treatment strategies based on the individual patient's condition. It is becoming increasingly evident that anticancer treatments, such as radiotherapy, conventional therapies, and targeted agents, totally or partially rely on the activation of immune reactions. The multiple functions of TAMs in tumor progression imply that the targeting of this group of immune cells may represent a novel immunotherapeutic strategy. Moreover, it may offer a new GC treatment option through immune system modulation, altering the tumor microenvironment to block tumor progression. Specifically, TAMs could be removed or limited in the tumor microenvironment, or M2 to M1 macrophage transformation could be encouraged (Biswas et al., 2013; Mantovani et al., 2014).

Despite our best efforts to conduct a comprehensive analysis of TAM polarization and patient survival, there were some shortcomings to the present study. First, diverse cutoff values were applied in the included articles, which may have had a negative impact on the observed precision of TAMs and their polarization subsets as prognostic factors in GC. Second, discrepancies between results were probably due to variations in the methodologies employed, with some studies not taking into account the influence of the markers used and the distribution of TAMs in different histologic locations in GC tissues. Third, as we only included articles published in English, language bias may have been present. Fourth, some HRs and $95 \%$ CIs were calculated using data derived from Kaplan-Meier survival curves. Fifth, the number of included articles was relatively small.

In conclusion, it is clear that TAMs demonstrate both pro- and anti-tumorigenic properties in GC. The present meta-analysis revealed that high levels of total infiltrating TAMs and M2 macrophages correlate with poorer OS in GC patients, and elevated M1 macrophage density may be regarded as a positive prognostic biomarker. However, increased numbers of total infiltrating TAMs showed no association with DFS of GC patients. Due to the limitations of the present analysis, further prospective studies employing a standardized methodology and larger sample sizes are needed to validate its conclusions. Moreover, investigations exploring the underlying mechanisms by which TAMs affect tumor progression would contribute to new GC therapy approaches.

\section{Conflicts of interest}

The authors declare no conflict of interest.

\section{ACKNOWLEDGMENTS}

Research supported by the National Key Technology R\&D Program of China (\#2015BAI12B12), the National Natural Science Foundation of China (\#31570877, \#31570908, and \#81171653), the Hong Kong, Macao and Overseas Scholars Cooperative Research Fund of the National Natural Science Foundation of China (\#31428005), and the Condition Construction and Livelihood Science and Technology Special Funds of Jiangsu Province (\#BL2014034).

\section{REFERENCES}

\footnotetext{
Amoueian S, Attaranzadeh A and Montazer M (2015). Intratumoral CD68-, CD117-, CD56-, and CD1a-positive immune cells and the survival of Iranian patients with non-metastatic intestinal-type gastric carcinoma. Pathol. Res. Pract. 211: 326-331.http://dx.doi.org/10.1016/j.prp.2014.12.013
}

Genetics and Molecular Research 15 (4): gmr15049040 
Balkwill F, Charles KA and Mantovani A (2005). Smoldering and polarized inflammation in the initiation and promotion of malignant disease. Cancer Cell 7: 211-217. http://dx.doi.org/10.1016/j.ccr.2005.02.013

Biswas SK and Mantovani A (2010). Macrophage plasticity and interaction with lymphocyte subsets: cancer as a paradigm. Nat. Immunol. 11: 889-896. http://dx.doi.org/10.1038/ni.1937

Biswas SK, Allavena P and Mantovani A (2013). Tumor-associated macrophages: functional diversity, clinical significance, and open questions. Semin. Immunopathol. 35: 585-600.http://dx.doi.org/10.1007/s00281-013-0367-7

Go Y, Tanaka H, Tokumoto M, Sakurai K, et al. (2016). Tumor-associated macrophages extend along lymphatic flow in the pre-metastatic lymph nodes of human gastric cancer. Ann. Surg. Oncol. 23 (Suppl 2): S230-S235.http://dx.doi. org/10.1245/s10434-015-4458-7

Gottfried E, Kunz-Schughart LA, Weber A, Rehli M, et al. (2008). Expression of CD68 in non-myeloid cell types. Scand. J. Immunol. 67: 453-463.http://dx.doi.org/10.1111/j.1365-3083.2008.02091.x

Haas M, Dimmler A, Hohenberger W, Grabenbauer GG, et al. (2009). Stromal regulatory T-cells are associated with a favourable prognosis in gastric cancer of the cardia. BMC Gastroenterol. 9: 65. http://dx.doi.org/10.1186/1471$\underline{230 X-9-65}$

Higgins JP and Thompson SG (2002). Quantifying heterogeneity in a meta-analysis. Stat. Med. 21: 1539-1558. http:// dx.doi.org/10.1002/sim.1186

Higgins JP, Thompson SG, Deeks JJ and Altman DG (2003). Measuring inconsistency in meta-analyses. BMJ 327: $557-$ 560. http://dx.doi.org/10.1136/bmj.327.7414.557

Ishigami S, Natsugoe S, Tokuda K, Nakajo A, et al. (2003). Tumor-associated macrophage (TAM) infiltration in gastric cancer. Anticancer Res. 23 (5A): 4079-4083.

Kawahara A, Hattori S, Akiba J, Nakashima K, et al. (2010). Infiltration of thymidine phosphorylase-positive macrophages is closely associated with tumor angiogenesis and survival in intestinal type gastric cancer. Oncol. Rep. 24: 405-415. http://dx.doi.org/10.3892/or 00000873

Kim KJ, Wen XY, Yang HK, Kim WH, et al. (2015). Prognostic implication of M2 macrophages are determined by the proportional balance of tumor associated macrophages and tumor infiltrating lymphocytes in microsatellite-unstable gastric carcinoma. PLoS One 10: e0144192. http://dx.doi.org/10.1371/journal.pone.0144192

Komohara Y, Jinushi M and Takeya M (2014). Clinical significance of macrophage heterogeneity in human malignant tumors. Cancer Sci. 105: 1-8. http://dx.doi.org/10.1111/cas.12314

Kübler K, Ayub TH, Weber SK, Zivanovic O, et al. (2014). Prognostic significance of tumor-associated macrophages in endometrial adenocarcinoma. Gynecol. Oncol. 135: 176-183.http://dx.doi.org/10.1016/j.ygyno.2014.08.028

Liberati A, Altman DG, Tetzlaff J, Mulrow C, et al. (2009). The PRISMA statement for reporting systematic reviews and meta-analyses of studies that evaluate healthcare interventions: explanation and elaboration. BMJ 339: b2700. http:// dx.doi.org/10.1136/bmj.b2700

Lin CN, Wang CJ, Chao YJ, Lai MD, et al. (2015). The significance of the co-existence of osteopontin and tumorassociated macrophages in gastric cancer progression. BMC Cancer 15: 128. http://dx.doi.org/10.1186/s12885-015$\underline{1114-3}$

Liu L, Ge D, Ma L, Mei J, et al. (2012). Interleukin-17 and prostaglandin E2 are involved in formation of an M2 macrophage-dominant microenvironment in lung cancer. J. Thorac. Oncol. 7: 1091-1100. http://dx.doi.org/10.1097/ JTO.0b013e 3182542752

Mantovani A and Locati M (2013). Tumor-associated macrophages as a paradigm of macrophage plasticity, diversity, and polarization: lessons and open questions. Arterioscler. Thromb. Vasc. Biol. 33: 1478-1483. http://dx.doi.org/10.1161/ ATVBAHA.113.300168

Mantovani A, Vecchi A and Allavena P (2014). Pharmacological modulation of monocytes and macrophages. Curr. Opin. Pharmacol. 17: 38-44.http://dx.doi.org/10.1016/j.coph.2014.07.004

Mills CD (2012). M1 and M2 macrophages: oracles of health and disease. Crit. Rev. Immunol. 32: 463-488. http://dx.doi. org/10.1615/CritRevImmunol.v32.16.10

Mills CD and Ley K (2014). M1 and M2 macrophages: the chicken and the egg of immunity. J. Innate Immun. 6: 716-726. http://dx.doi.org/10.1159/000364945

Murray PJ and Wynn TA (2011). Protective and pathogenic functions of macrophage subsets. Nat. Rev. Immunol. 11: 723-737. http://dx.doi.org/10.1038/nri3073

Nam SJ, Go H, Paik JH, Kim TM, et al. (2014). An increase of M2 macrophages predicts poor prognosis in patients with diffuse large B-cell lymphoma treated with rituximab, cyclophosphamide, doxorubicin, vincristine and prednisone. Leuk. Lymphoma 55: 2466-2476. http://dx.doi.org/10.3109/10428194.2013.879713

Ohno S, Inagawa H, Dhar DK, Fujii T, et al. (2005). Role of tumor-associated macrophages (TAM) in advanced gastric carcinoma: the impact on FasL-mediated counterattack. Anticancer Res. 25 (1B): 463-470.

Genetics and Molecular Research 15 (4): gmr15049040 
Park JY, Sung JY, Lee J, Park YK, et al. (2016). Polarized CD163+ tumor-associated macrophages are associated with increased angiogenesis and CXCL12 expression in gastric cancer. Clin. Res. Hepatol. Gastroenterol. 40: 357-365. http://dx.doi.org/10.1016/j.clinre.2015.09.005

Pei BX, Sun BS, Zhang ZF, Wang AL, et al. (2014). Interstitial tumor-associated macrophages combined with tumorderived colony-stimulating factor-1 and interleukin-6, a novel prognostic biomarker in non-small cell lung cancer. $J$. Thorac. Cardiovasc. Surg. 148: 1208-1216.e2.http://dx.doi.org/10.1016/j.jtcvs.2014.05.003

Reinartz S, Schumann T, Finkernagel F, Wortmann A, et al. (2014). Mixed-polarization phenotype of ascites-associated macrophages in human ovarian carcinoma: correlation of CD163 expression, cytokine levels and early relapse. Int. J. Cancer 134: 32-42. http://dx.doi.org/10.1002/ijc.28335

Ruffell B and Coussens LM (2015). Macrophages and therapeutic resistance in cancer. Cancer Cell 27: 462-472. http:// dx.doi.org/10.1016/j.ccell.2015.02.015

Sica A, Larghi P, Mancino A, Rubino L, et al. (2008). Macrophage polarization in tumour progression. Semin. Cancer Biol. 18: 349-355. http://dx.doi.org/10.1016/j.semcancer.2008.03.004

Stang A (2010). Critical evaluation of the Newcastle-Ottawa scale for the assessment of the quality of nonrandomized studies in meta-analyses. Eur. J. Epidemiol. 25: 603-605. http://dx.doi.org/10.1007/s10654-010-9491-z

Thrumurthy SG, Chaudry MA, Chau I and Allum W (2015). Does surgery have a role in managing incurable gastric cancer? Nat. Rev. Clin. Oncol. 12: 676-682. http://dx.doi.org/10.1038/nrclinonc.2015.132

Tierney JF, Stewart LA, Ghersi D, Burdett S, et al. (2007). Practical methods for incorporating summary time-to-event data into meta-analysis. Trials 8: 16. http://dx.doi.org/10.1186/1745-6215-8-16

Torre LA, Bray F, Siegel RL, Ferlay J, et al. (2015). Global cancer statistics, 2012. CA Cancer J. Clin. 65: 87-108. http:// dx.doi.org/10.3322/caac. 21262

Wang B, Xu D, Yu X, Ding T, et al. (2011). Association of intra-tumoral infiltrating macrophages and regulatory T cells is an independent prognostic factor in gastric cancer after radical resection. Ann. Surg. Oncol. 18: 2585-2593. http:// dx.doi.org/10.1245/s10434-011-1609-3

Wu MH, Lee WJ, Hua KT, Kuo ML, et al. (2015). Macrophage infiltration induces gastric cancer invasiveness by activating the beta-catenin pathway. PLoS One 10: e0134122.http://dx.doi.org/10.1371/journal.pone.0134122

Yuan ZY, Luo RZ, Peng RJ, Wang SS, et al. (2014). High infiltration of tumor-associated macrophages in triple-negative breast cancer is associated with a higher risk of distant metastasis. Onco Targets Ther. 7: 1475-1480. http://dx.doi. org/10.2147/OTT.S61838

Zhang H, Wang X, Shen Z, Xu J, et al. (2015). Infiltration of diametrically polarized macrophages predicts overall survival of patients with gastric cancer after surgical resection. Gastric Cancer 18: 740-750. http://dx.doi.org/10.1007/ $\underline{\text { s10120-014-0422-7 }}$

Zhang J, Yan Y, Yang Y, Wang L, et al. (2016). High infiltration of tumor-associated macrophages influences poor prognosis in human gastric cancer patients, associates with the phenomenon of EMT. Medicine (Baltimore) 95: e2636. http://dx.doi.org/10.1097/MD.0000000000002636

Zhang QW, Liu L, Gong CY, Shi HS, et al. (2012). Prognostic significance of tumor-associated macrophages in solid tumor: a meta-analysis of the literature. PLoS One 7: e50946. http://dx.doi.org/10.1371/journal.pone.0050946

Zhang Y, Cheng S, Zhang M, Zhen L, et al. (2013). High-infiltration of tumor-associated macrophages predicts unfavorable clinical outcome for node-negative breast cancer. PLoS One 8: e76147. http://dx.doi.org/10.1371/ journal.pone. 0076147

Genetics and Molecular Research 15 (4): gmr15049040 